\title{
Nanogels and microgels: The new polymeric materials playground
}

\author{
Neil B. Graham and Audrey Cameron \\ Department of Pure and Applied Chemistry University of Strathclyde, Glasgow, Scotland, UK
}

\begin{abstract}
Microgels, or intramolecularly crosslinked macromolecules (ICMs), have been known for a number of years. They are formed during the polymerization of polyfunctional precursors en-route, but at incomplete reaction, to the macrogelation predicted by the theories of gelation initially propounded by Carothers and Flory. These gelation theories did not predict that such microgels made in solution could be prepared from such polyfunctional reactive solutions at complete reaction and high concentrations, without gelation. Microgels have been successfully and usefully prepared, however, at complete conversion of their contained reactive groups by the use of either aqueous or non-aqueous dispersion polymerization in which surface active agents are utilized to contain the polymerization to isolated submicron-size domains. The progression towards macrogelation is constrained to the maximum size of the dispersed domains. This paper demonstrates the contrasting general observation that macrogelation in solvents of such polyfunctional reactive precursors cannot occur below a Critical Gelation Concentration (CGC) if a carefully selected match of solvent solubility parameters for the polymerization solvent and the formed polymer is made. In some cases the addition of only ca. ten percent $\mathrm{w} / \mathrm{w}$ of solvent is required to completely prevent macrogelation at complete conversion. In addition polymerization the molecular weight of the produced ICMs may be varied in a simple controllable manner from the low thousands (nanogels) to many millions (microgels) simply by the choice of concentration at which they are prepared. The products are a distinct form of polymer which is quite different to the linear analogue and almost certainly these solution-prepared products are different to the microgels formed by aqueous dispersion polymerization techniques. This new solution technique provides a simple and general new method for the ready synthesis and design of an enormous range of bespoke globular polymers having both fundamental academic interest and potential commercial utility.
\end{abstract}

\section{INTRODUCTION}

Bifunctional monomer combinations which can polymerize without chain branching form linear polymers which can attain extremely high molecular weight into the millions. Melts of such high molecular weight polymers and even very low concentration solutions of them can have very high viscosity. Their ability to be fabricated or utilized is thus very restricted so a compromise utilizing lower than optimum molecular weights is common. Monomer combinations even slightly in excess of two form macrogelled networks when polymerized in bulk. Such macrogelled networks retain the covalent network shape in which they were initially manufactured as a permanent memory. They do not flow in the presence of solvents and cannot be dissolved without the breaking of covalent bonds. Their fabrication is also difficult though widely used in paints, thermosets and laminates. As many of their properties are highly desirable, new forms of these materials and improved methods for their fabrication are very worthwhile objectives. This paper describes a new set of procedures for the simple solution preparation of soluble crosslinked Intra Molecular Crosslinked Macromolecules (ICMs) having molecular weights in the range from a few tens of thousands to many millions. There does not appear to be any restriction on the type of polymer or polymerization mechanism which can be utilized. The study of this type of process is leading to a greater understanding of the progress of the overall gelation process in terms of different types of ICMs which have been classified herein as; nanogel and microgel. At complete reaction of the functional groups initially present the product can be any one of these two types of ICM or a macrogel which may itself have many potentially different structures and properties. 


\section{MICROGEL FORMATION HYPOTHESIS}

The term microgel was coined by Baker (ref. 1) who from work on emulsions of GRS or SBR rubbers recognized that these cross-linked structures were Intramolecularly Crosslinked Macromolecules which constituted a new form of polymer molecule. He proposed the name Microgel for these. The name stuck along with the recognition of their importance as a distinct variety of polymeric structure and material. In his examination of their properties he demonstrated that for their very large size they showed very low solution viscosities and the molecular weight exponent in the Mark-Houwink equation was very low $(<0.5)$ and in some instances approached zero. This demonstrated that in "solution" these microgels behaved more like Einstein spheres than dissolved linear polymer coils and the microgels exhibited much lower than expected solution viscosities. It is thus not surprising that these condensation ICMs or microgels became recognized by Bobalek in particular, as important contributors to the properties of alkyd resins in the paint industry. It was not possible at this time to make microgels at complete reaction of the components of a polyfunctional polymerization. They could not be readily made, isolated and studied. Later work by Funke demonstrated how in a manner similar to the original preparation of GRS or ABS emulsion polymerization provided a practically applicable route to the preparation of microgels having the non-swollen size of the latex particle. These preparations usually required the use of surface-active agents. Some surfactant-free microgels utilizing copolymerized acids (ref. 2) which also acted as emulsion stabilizers were made. This method of preparation has its difficulties and limitations.

Our hypothesis for the preparation of microgels was that in a good solvent for the polymer to be formed, it should be possible to polymerize a monomer mixture having an overall functionality of greater than two without macrogelation. At some concentration in the optimum solvent (combination) the polymerization should always form soluble microgels and not an infinite macrogel. The macrogel, if formed at all, must be formed through the prior formation of microgel. Microgel however will normally contain dead chains, polymerizing chains and loops of polymer all of which can act as very effective steric stabilizers in a good solvent (refs. 3,4). These chains should prevent contact between the microgel particles and largely prevent particle-particle reaction and combination while promoting the intramolecular crosslinking. This hypothesis has been tested by polymerizing a wide range of crosslinking systems in a wide range of solvents of graduated solubility parameter and hydrogen bonding class over a range of concentrations (w/v) from 2$95 \%$ (refs. 5,6,7).

\section{Verification of the Hypothesis for Addition Polvmerization}

The experiments illustrated here are a comparison of the microgel formation of methyl methacrylate(MMA) and hydroxyethyl methacrylate(HEMA) crosslinked using diethyleneglycol dimethacrylate(DEGDMA). A typical illustrative recent set of experiments is shown for the MMA series in Table 1. The pattern was repeated for HEMA. Microgels were formed within the perimeter of a Critical

TABLE 1. Methyl methacrylate - Diethyleneglycol dimethacrylate $95 \% \mathrm{w} / \mathrm{w} \mathrm{MMA}+5 \% \mathrm{w} / \mathrm{w}$ DEGDMA (4 pph Benzoyl peroxide) at $80^{\circ} \mathrm{C}$ for one week.

\begin{tabular}{|cccccc|}
\hline Code No. & Solvent & $\begin{array}{c}d \\
\text { cal }^{1 / 2} \mathbf{c m}^{-3 / 2}\end{array}$ & $\% \mathbf{M}$ & $\%$ Conversion & System gelled? \\
11A,B,C & Ethyl caprylate & 7.3 & $5,10,20$ & - & Yes \\
12A,B,C & 5-Nonanone & 8.0 & $5,10,20$ & - & Yes \\
13A & 3-Pentanone & 8.8 & 5 & 95.1 & No \\
13B & 3-Pentanone & 8.8 & 10 & 100 & No \\
13C & 3-Pentanone & 8.8 & 20 & 100 & No \\
14A & 2-Butanone & 9.3 & 5 & 92.9 & No \\
14B & 2-Butanone & 9.3 & 10 & 100 & No \\
14C & 2-Butanone & 9.3 & 20 & 100 & No \\
15A & $95 \%$ Acetone + 5\% Water & 10.6 & 5 & 94.7 & No \\
15B & $95 \%$ Acetone + 5\% Water & 10.6 & 10 & 97.7 & No \\
15C & $95 \%$ Acetone + 5\% Water & 10.6 & 20 & 100 & No \\
16A,B,C & $75 \%$ Acetone + 25\% Water & 13.3 & $5,10,20$ & - & Yes \\
17A,B,C & $50 \%$ Acetone + 50\% Water & 16.7 & $5,10,20$ & - & Yes \\
\hline
\end{tabular}


Gelation Concentration (CGC) curve illustrated for the MMA and HEMA series in Figs 1 and 2. The maximum initial monomer concentrations for successful microgel preparations were attained at different solubility parameter values and hydrogen-bonding characteristics for each series as expected. Hydroxyethylmethacrylate (HEMA) requires strongly hydrogen-bonding solvents for successful microgel preparation. In many studies using a wide variety of monomers we have never yet failed in the successful preparation of soluble ICMs by the selection of the appropriate solvent solubility parameter.
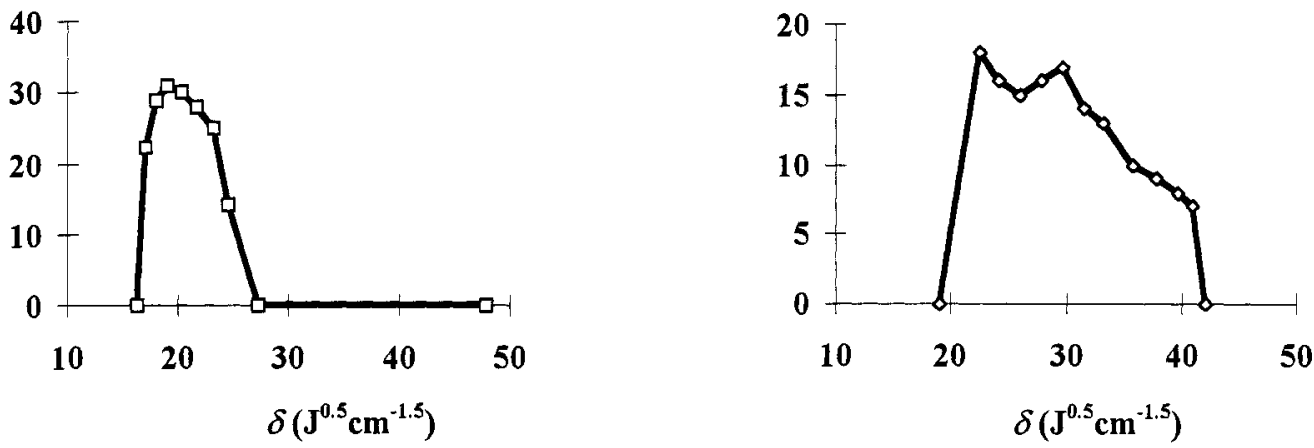

Fig. 1 MA/DEGDME 95/5 Polymerisations at Fig. 2 HEMA/DEGDME 90/10 Polymerisations $80^{\circ} \mathrm{C}$. Plot of CGC against solubility parameter $(\delta)$ at $60^{\circ} \mathrm{C}$. Plot of CGC against solubility parameter of the solvent used for the polymerization

$(\delta)$ of the solvent used for the polymerization

The determination of the molecular weights $\left(M_{n}\right.$ or $\left.M_{w}\right)$ is a most important characterization for these materials but it is impossible to obtain even approximately accurate values with the conventional size exclusion chromatography or gel permeation chromatography (GPC) now used in most polymer laboratories. Trends and relative values between different microgels do have meaning however using GPC. Absolute techniques are required for reliable values and the combination of GPC with light scattering is particularly useful. The light scattering molecular weight averages are usually at least five time greater than those obtained from the GPC using conventional linear polymer calibration standards. Values quoted in this paper are those from GPC unless otherwise stated and must be treated with caution and recognized as approximately one-fifth of their correct values. Table 2 below shows some GPC molecular weights which change dramatically with the concentration of preparation. A number of points bear comment. The first is that the molecular weight of the microgels can be changed from much less than an $M_{w} 100,000$ to many millions (shown by light scattering) by the simple change of monomer concentration. The microgel polymers of many millions molecular weight are, perhaps surprisingly still free flowing solutions even when quite concentrated. The $M_{n}$ values of even the highest molecular weight microgels remain low and even when the $M_{w}$ increases dramatically $M_{n}$ does not increase to a major degree. These low $M_{n}$ values indicate the continuing presence of a significant proportion of low molecular weight ICMs which may be shown to have a radius of gyration of less than ten $\mathrm{nm}$ in solution. They are thus more appropriately called nanogels and will be referred to in this way subsequently. The nanogels are formed exclusively below certain concentrations which appear to correspond to solutions in which the solvent swollen particles are not yet at a high enough concentration to force them into intimate contact at their surfaces. The swollen coil densities of these nanogel and microgel particles at infinite dilution can be estimated from their intrinsic viscosities using the Einstein-Stokes equation. At a polymerization concentration of $20 \%$, it can be seen that the swollen densities are high enough to force the fully converted and solvent swollen nanogel particles into full occupation of the available volume and so into intimate surface contact. The increasingly high molecular weight polymer begins to form with a dramatic increase in the heterogeneity index beyond $20 \% \mathrm{w} / \mathrm{w}$ polymerizing monomer concentration as illustrated by the results for MMA/DEGDMA in Fig. 3. In spite of the very close forced approach of the nanogel particle surfaces, amazingly, no macrogelation occurs and soluble mixtures of nanogel and microgel are formed in "solution" up to much higher concentrations.

\section{Verification of the Hypothesis for Step-growth Polymerization}

Studies of the preparation of polymers from poly(ethylene glycol) (PEG), the triol 1,2,6-hexane diol and stoichiometric proportions of biscyclohexylmethane- $4,4^{\prime}$-diisocyanate in various ratios of the solvent methyl 
ethyl ketone as an example of a step-growth polymerization demonstrated that it was impossible to cause macrogelation of the system below Critical Solution Concentrations which were a specific characteristic of each solvent system. For a PEG of nominal $\mathrm{M}_{\mathrm{n}} 6000$, a $\mathrm{CGC}$ of the incredibly high value of $93 \% \mathrm{w} / \mathrm{w}$ reactants/2-butanone was obtained at complete reaction. The products were nanogels of light scattering (GPC/RALS) $M_{n} 77000$ and $M_{w} 129900$ with a heterogeneity index of 1.69. It proved almost impossible to form very high molecular weight polymers by this solution technique and a special preparative method had to be developed for such high molecular weight materials. The generality of this nanogel/microgel preparative procedure has thus been demonstrated. It has been confirmed (ref. 8) for other polyfunctional systems utilizing polymerization mechanisms such as cationic.

\section{Crystalline ICMs}

It is of interest that the low and the high molecular weight PEG nanogels and microgels synthesized by the above methods were highly crystalline in the solid state and could be readily crystallized from solution as spherical granules of controllable granule sizes. These can be readily melted, molded or powder compacted into potentially useful controlled drug delivery systems $($ refs 9,10$)$ of particular use for high molecular weight actives which are normally difficult to formulate for sustained and programmed delivery.

TABLE 2. Molecular weights, intrinsic viscosities and solution coil densities of nanogels, microgels and linear polymers of MMA or MMA/DEGDME $95 / 5 \mathrm{w} / \mathrm{w}$ made in 2-butanone at $80^{\circ} \mathrm{C}$

\begin{tabular}{|l}
$\begin{array}{l}\text { Monomere } \\
\text { concentrationrereference } \\
\text { (\%w/w) }\end{array}$ (GPC) \\
Nanogels and microgels
\end{tabular}

Fig. 3 The heterogeneity index variation with the monomer concentration of preparation for MMA/DEGDMA at $80^{\circ} \mathrm{C}$ in 2-butanone 


\section{$\underline{\text { Loopy molecules }}$}

All of the nanogels and microgels described in this paper of necessity contain loops and rings within their molecular structures. The molecule of $\mathrm{M}_{\mathrm{n}} 77000$ based on the cross linking of PEG 6000 can only contain approximately ten such rings and have structures which are not unduly complex with the size of the formed rings being limited to very specific multiples of ca. 6000 .

This also applies to the crosslinked low molecular weight nanogels prepared from vinyl monomers in which the number of rings are also low in number though in this case the size of the rings will be less well defined. The molecular weights of both of these classes of materials can be reduced by the addition of chain terminators or chain transfer agents and it would appear that the synthesis of designer molecules containing from one to any number of rings may be attainable via this synthetic route. Even without such a degree of sophistication this synthetic process offers the broad opportunity to synthesize a great range of polyfunctional soluble ICMs which have potential in many different materials applications. In our hands they are proving especially useful for the design of so-called "Smart Systems".

\section{REFERENCES}

1. W.O. Baker, Ind. Eng. Chem., 41, 511, (1949)

2. W. Funke, et. al., Die Angewandte Makromolekulare Chemie, 103, 187-202, (1982)

3. Dispersion Polymerisation in Organic Media, Ed. K.E.J. Barrett, John Wiley and Sons, (1975)

4. Polymeric stabilisation of colloidal dispersions, Ed. D.H. Napper, Academic Press (1983)

5. N.B. Graham and Carmel M.G. Hayes, Macromolecular Symposium, Polymer Networks 1994, 93, 293 $300,(1995)$

6. N.B. Graham, Colloids and Surfaces A: Physicochemical and Engineering Aspects, 118, 211-220, (1996)

7. N.B. Graham, J. Mao and A. Urquhart, Die Angewandte Makromolekulare Chemie, Applied Macromolecular Chemistry and Physics, 240, 113-121, (1996)

8. N.B. Graham, Polymerisation Process, UK Patent 2090264, filed December 1981, issued October 1984

9. N.B. Graham, and Jianwen Mao, 52-64 in Chemical Aspects of Drug Delivery Systems, Eds. D.R. Karsa, and R.A. Stephenson, The Royal Society of Chemistry, (1996)

10. J. Mao, Polyurethane Microgels and Controlled Drug Delivery, PhD Thesis, University of Strathclyde, Glasgow, Scotland, UK, (1995) 